\title{
On the application of frequency tagging
}

\author{
Zoltan Derzsi \\ ha5dzs@gmail.com
}

\section{Abstract}

5 To detect a weak signal in human electrophysiology that is a response of a periodic external stimulus, spectral evaluation is mostly used. The recorded signal's amplitude and phase noise components of the signal are statistically independent from each other, but both of them are decreasing the signal-to-noise ratio, which results in a lower probability of successful signal detection. Provided that the phase information of the stimuli is preserved, we found that a way to reject an additional phase noise component, which improves the detection probability considerably, by analysing the signal's phase coherency instead of its spectrum.

\section{Introduction}

In many cases across various fields of electrophysiology, an experiment with a living organism involves the recording of electrical signals. The difficulty of acquiring a clean, high quality recording can not be overstated: many recorded signals are quite weak, in the millivolt or perhaps even in the microvolt range. At these low voltages, the noise might well be stronger than the actual signal itself, which may render a set of collected data useless, and a subsequent analysis on this data inconclusive. A logical approach to counter-act the effect of noise would be to use appropriate filters, de-noising algorithms on the data or perhaps eliminating or suppressing external noise sources 
while recording if possible.

If we consider the living organism responding to a periodic stimulus as the signal source or transmitter, and the data recorder as the receiver, we could say that the statistical evaluation of the data is the process of signal detection. In practice, when a signal amplitude is beyond reasonable doubt (i.e. the P-value) distinct from the noise, we could say that the signal was successfully detected.

A simple implementation of this is the study of event-related-potentials (ERPs). For example, when a human sees a camera flash going off, a transient can be observed in the recorded electroencephalograph (EEG) signal [Ciganek, 1961]. The signal is usually very weak, so the recordings were segmented for each trial and then temporally aligned to the flashes, and averaged afterwards. These 'flash-evoked potentials' were then analysed in the time domain.

It is possible to present these flashes repeatedly at a particular temporal frequency, which would also create a repeated peaks in the EEG signal with the same temporal frequency. It is also possible to present multiple flashes at different temporal frequencies and then analyse the intermodulational products (sum and difference of these frequencies) in the EEG signal. The technique employing these temporally generated or modulated stimuli is known as frequency-tagging.

\section{Why use frequency tagging?}

Following on from the transmitter-receiver analogy where the transmitter is a stimulus which is being modulated at a known temporal frequency; the living organism being tested is the medium in which the signal propagates and usually greatly varies, and the receiver is the recording equipment. This is quite similar in spirit to receiving distant shortwave broadcast radio stations, where the transmission quality changes heavily with how well the Earth's ionosphere reflects radio waves.

The term 'frequency tagging' is one name to describe this technique: the stimulus may be modulated with one or many temporal frequencies, and the presence and 
interaction of these 'tagged' frequencies or their harmonics are observed in the recording. This stimulus may be as simple as flashing lights [Baitch and Levi, 1988] or faces [Boremanse et al., 2013] presented in the visual field in humans, but the technique has been demonstrated to work with tactile stimuli as well [Moungou et al., 2016]. It also works with more advanced stimuli: when studying human depth perception, particularly stereopsis, frequency tagging is often the preferred method [Norcia and Tyler, 1984, Baitch and Levi, 1988, Boremanse et al., 2013, Cottereau et al., 2014, Norcia et al., 2015]. We tested the spatio-temporal limits of human stereopsis by using dynamic correlated random-dot stereograms [Julesz, 1971]. These are stimuli that only contain depth information, and they look like noise in a de-tuned analogue television set if someone looks at it with one eye closed. While the participants looked at our 3D stimuli, we recorded their electroencephalography (EEG) signals, and organised the presentation of the stimuli in short trials. We presented a single plane made out of depth, which jumped in front of and behind the screen plane. This condition was very similar to what Norcia and Tyler used in [Norcia and Tyler, 1984], and their stimulus was illustrated in figure 1. The depth alternation frequency was set to $2.1 \mathrm{~Hz}$ and was always in the same phase across the trials.

We then analysed the signal. We have aligned these trials to the onset of the alternating visual stimulus, and (1) we averaged across the trials in the frequency domain, and (2) we calculated the inter-trial phase coherency [Norcia and Tyler, 1984] of the signal.

The spectra of the trials did not contain the frequency-tagged signal elicited by our stimulus. However, the coherency analysis of the same signal revealed that there are indeed traces of the signal within our recordings: the second, fourth, and even the sixth harmonics have been successfully detected, much to our surprise. As shown in figure 2 , the advantage of coherency analysis is apparent.

In this paper, we will show that not all detection methods perform equally well when the conditions are noisy, and that the detection sensitivity can be greatly improved with 
coherency analysis, provided that the phase information of the stimulus was known. With the use of this technique, it is possible to reduce the number of trials needed, or to detect weak signals that other methods would fail to find. We believe that the signal detection method described in this paper will help making frequency tagging an even more powerful method, and the signal detection practices may be applicable to other areas where weak-signal communication is required.

\section{Methods}

Irrespective of the field and the stimulus type frequency tagging is used in, it always involves some form of time-frequency transform to analyse the spectral component corresponding to the harmonics of the temporal frequency of the stimulus. This transform is usually the Fourier-transform, but there are analysis methods readily available in free software packages such as EEGLAB [Delorme and Makeig, 2004] which use the wavelet transform [Daubechies, 1990]. To improve the probability of detection, a large number of trials are included in the analysis, in order to increase the signal-to-noise ratio (SNR). In practice, with human EEG, this can mean hundreds of trials, for a signal that can be so weak that it's several hundred times below the noise level.

The captured and averaged signals are analysed using their spectral power levels of the corresponding frequencies. To determine whether the power levels are significantly increased, a statistical analysis is carried out, effectively implementing the operation of the 'ideal receiver' as it is described in communication engineering textbooks [Proakis, 2000] [Proakis and Salehi, 2008].

\section{Inter-trial coherency analysis}

The spectral analysis described above uses the magnitudes of the spectral components. The rationale for this analysis method is that the stimulus elicits a 
response that results in a significantly increased power level at the frequency in question. If the detection is successful, the temporally modulated stimulus elicits a response where the power of the frequency component in question follows the same modulation pattern: for example, when flashes with two different temporal frequencies are present, the recorded signal may show significantly elevated power levels at the sum and difference of the two frequencies

[Baitch and Levi, 1988, Boremanse et al., 2013]. This is, in essence, a form of amplitude modulation: if the magnitudes of the spectral components are elevated and it can not be explained by chance alone, we can conclude that the signal has been detected successfully.

However, enclosing information in the amplitude of the signal is one of many (albeit the oldest and simplest [Proakis, 2000]) methods. It is also possible to enclose information in the phase of the signal: hypothetically, for example, if we started the visual stimuli of [Boremanse et al., 2013] in the exact same sequence at the beginning of the trial, the phase of both Fourier-components corresponding to the temporal frequencies of the visual stimulus would be the same across the trials. Therefore, when analysing the EEG recordings of these trials, we would find that not only the amplitudes are significantly increased, but the phase angles of these components would be similar from trial to trial as well.

Taking it one step further, it is possible to analyse the phase angles alone, effectively ignoring the amplitude component. The inter-trial coherency is a measure that expresses how consistent these phase angles are across the trials: if the phase angles are perfectly aligned which indicates a very strong signal, the coherency value is 1 , and when the phases are completely random across the trials, the coherency value is 0 [Tyler and Kaitz, 1977, Norcia and Tyler, 1984], indicating that there is no distinction from white noise. It is also worth noting that the coherency analysis ignores the phase value itself. As shown in figure 3, effectively, the coherency is a measure of how varied the phase angles are across the trials, as opposed to what values the phase angles are themselves in each trial. 


\section{Results}

\section{Signal-to-noise ratio and detection probability}

For a given SNR, and $L$ number of trials, it is possible to estimate the probability of erroneously detecting the signal. This number is the same that is used as significance indicators when presenting data in studies:

$$
P_{\text {spectral }}=\frac{1}{2} \operatorname{erfc} \sqrt{\frac{1}{2} \times \mathrm{SNR} \times \sum_{n=1}^{L}\left|g_{n}\right|^{2}}
$$

$$
P_{\text {coherency }} \approx \frac{1}{2} \operatorname{erfc} \sqrt{2 \times \mathrm{SNR} \times \sum_{n=1}^{L}\left|g_{n}\right|^{2}}
$$

...where $\operatorname{erf} c$ is the complementary error function, and $g_{n}$ is the gain of the system. In an experiment, the $g_{n}$ of the mechanism is what we are trying to characterise: this gain is determined by the physiology of the living organism, and will respond differently when the simulus conditions change. For human stereopsis, it is our estimate that $g_{n}$ converges to 0 when the depth plane stimuli reaches the alternation frequency of around $5-6 \mathrm{~Hz}$.

We can see from these equations that for the same SNR, and same number of trials, coherency analysis gives lower P-values, indicating that the coherency analysis is a more sensitive method for detecting the signal. The improvement stems from the rejection of the amplitude information in the received signal, which means that its noise component gets rejected as well. This is detailed across several chapters in engineering textbooks such as [Proakis and Salehi, 2008], and there is a brief explanation and proof tailored for the frequency-tagging application along with an explanation on why it is only an approximation for the coherency analysis in the appendix. 


\section{Simulation}

We have also created a simulation of a frequency tagging experiment. We created a weak signal that is always in the same phase in each trial, added noise, and analysed the data. The simulation had the following parameters:

- $f=15 \mathrm{~Hz}$, always in the same phase during the trials

- $\mathrm{SNR}=\frac{1}{300}$

- Number of trials: 150 and 2000

To detect the signal that is three hundred times weaker than the noise, we replicated the operation of the ideal receiver's behaviour. In this case it is an unpaired, single-tailed t-test at the $15 \mathrm{~Hz}$ frequency with respect to a distribution clearly built up by the noise, executed on both the spectrum and the coherency. At 150 trials, the spectral analysis did not produce significant $(P=0.413)$ results implying that the signal could not be detected, whereas the coherency analysis produced a P-value less than $10^{-4}$, with the signal clearly visible in figure 4 . We continued adding trials in the simulation, and at 2000 trials, both methods detected the signal, which is shown in figure 5 .

\section{Minimum number of trials required for successful detection}

For the spectral evaluation and the coherency analysis, our finding suggests that -ignoring the $g_{n}$ gain of the system being tested for the sake of simplicity- the minimum number of trials required to achieve a $P=0.05$ can be calculated as a function of SNR is:

$$
L_{\text {spectral }}=\frac{\left.\left[e r f c^{-1}(2 \times 0.05)\right]^{2}\right]}{\mathrm{SNR}}
$$

$$
L_{\text {coherency }} \approx \frac{\left.\frac{1}{4} \times\left[\operatorname{erfc}^{-1}(2 \times 0.05)\right]^{2}\right]}{\mathrm{SNR}}
$$

As the SNR decreases, all analysis methods require more trials to detect the frequency-tagged signals. Under extremely bad conditions which we assume to be 
typical for electrophysiology experiments, the signal is several hundred times below the noise level. To detect the signal, hundreds of trials may be required. For coherency, approximately, only a quarter of the number of trials are needed to detect the signal at the same significance level when compared to spectral evaluation. For a range of signal-to-noise ratios, the required trials to detect the signal at a $P=0.05$ significance level are shown in figure 6.

For our simulation, the minimum number of trials required is around 140 trials when using the coherency analysis, and around 530 trials when using spectral analysis. Using more trials will decrease the P-values.

In order to keep the findings more robust, more than the minimum required trials may be used, since in practice the SNR in each experiment can only be estimated and can change stochastically.

\section{Conclusion}

We have proven that the inter-trial coherency analysis is a better method for analysing frequency-tagged data. We found that, provided that the phase as well as the frequency information is preserved in an experiment employing the frequency tagging method, it is possible to detect an approximately four times weaker signal with the same amount of trials in the coherency analysis when compared to the spectral analysis. Conversely, the same signal can be detected with approximately a quarter of the number of trials with the coherency analysis than the spectral analysis frequently used. The simulation results with a weak signal and white noise are consistent with real-life EEG measurements on human stereopsis.

Therefore, based on our results, we recommend keeping the phase of the frequency tagging signal known across trials, and not changing the number of trials used in the experiment. With the coherency analysis, it is possible to create a signal detector that is capable of capturing very weak signals that would not be possible otherwise. 


\section{Acknowledgements}

We would like to thank the Wellcome Trust for funding the project, and NVIDIA for providing a high-performance graphics processing unit that helped us with the simulations.

\section{Appendix}

\section{Noise components in the detected signal}

Every received signal has added noise, this is unavoidable. Depending on the technique used, there can be a number of noise sources in the environment in which the signal was recorded. However, at the receiver, from the frequency tagging point of view, since the frequency of the stimulus are known, the majority of the noise can be eliminated simply by rejecting all frequency components that are known to be unrelated to the stimulus. The rejection can be done by using precisely tuned discrete filters, as was done in [Norcia and Tyler, 1984], or by using a time-frequency transform and selecting the appropriate coefficients, as it was done in subsequent studies, such as [Boremanse et al., 2013].

The received signal will then expressed as $R(t)=\left(\sqrt{P_{b}}+d \sqrt{P_{b}} d t\right) \times\left(\cos \omega_{S} t+d \varphi d t\right)$, which is effectively a noisy sine wave with the power $\sqrt{P_{b}}$ for each selected frequency $\omega_{S}$. The noise components that are added to the received signal are expressed as $d \sqrt{P_{b}}$ and $d \varphi$. These noise components are known to be additive and statistically independent from each other [Proakis, 2000], with their effect shown in figure 8. The delivered energy $d \sqrt{E_{b}}$ changes the length of the vector and shows the effect of the amplitude noise, and $d \varphi d t$ moves the vector's end along the indicated tangential line, showing the effect of the phase noise. 


\section{Detection methods for a single trial}

On the assumption that the phase of the frequency-tagged signal is known, it is possible to cancel out the $d \sqrt{E_{b}}$ term in the received signal, effectively improving the signal-to-noise ratio, and thus making the detection more reliable. The following approach calculates the error probability of a binary answer of the signal detector. If we treat the frequency-tagged signal as a carrier with phase modulation, the probability of false detection can be derived as follows [Proakis, 2000, Proakis and Salehi, 2008]: Since we have established that the received signal has a vector representation, and the frequency tagging is used to investigate the behaviour of a system under test, it is possible to say that:

$$
R S(t)=g(t)\left[2 \pi f_{c}(t)+\pi\right]
$$

$R S(t)$ is now the received signal, $f_{c}$ is the carrier frequency, and $g(t)$ is the gain of the system under test. The gain can arbitrarily change, in the same manner how fading is experienced while receiving a distant shortwave radio broadcast, or how the EEG electrodes dry out over time for example. By the time the signal arrives to the receiver, it had already passed through the system under test, and has been subject to additive noise, which is assumed to be Gaussian as it's a natural system. The ideal receiver computes the correlation metrics that assign which signal states belong to which output value in the receiver [Proakis, 2000]:

$$
C_{r, R S(t)}=r \cdot R S(t)
$$

The message $r$ can be de-composed to statistically independent components $r=\left[\begin{array}{ll}r_{1} & r_{2}\end{array}\right]$, and the phase angle which contains the information is obtained:

$$
\varphi=\arctan \frac{r_{2}}{r_{1}}
$$

Let's assume that the received signal's phase angle is $\varphi=0^{\circ}$. The vector 


$$
\begin{aligned}
& r_{1}=\sqrt{E_{b}}+n_{1} \\
& r_{2}=n_{2}
\end{aligned}
$$

...where $n_{1}$ and $n_{2}$ are two noise components. This gives two Gaussian distributions at the receiver, with identical standard deviations. In particular, these are expressed as:

$$
\begin{aligned}
E\left(r_{1}\right) & =\sqrt{E_{b}} \\
E\left(r_{2}\right) & =0 \\
\text { and } \sigma_{r_{1}}^{2} & =\sigma_{r_{2}}^{2}=\sigma_{r}^{2}=\frac{1}{2} N_{0}
\end{aligned}
$$

$\ldots$ where $N_{0}$ is the noise energy in the single trial. The message's probability distribution function can then be expressed as [Proakis, 2000]:

$$
P_{r}\left(r_{1}, r_{2}\right)=\frac{1}{2 \pi \sigma_{r}^{2}} \exp \left[-\frac{\left(r_{1}-\sqrt{E_{b}}\right)^{2}+r_{2}^{2}}{2 \sigma_{r}^{2}}\right]
$$

and since we know that the receiver's output value is $V=\sqrt{r_{1}^{2}+r_{2}^{2}}$, we can:

$$
P_{V_{r}, \varphi}\left(V_{r}, \varphi\right)=\frac{V}{2 \pi \sigma_{r}^{2}} \exp \left(-\frac{V^{2}+\sqrt{E_{b}}-2 \sqrt{E_{b}} V \times \cos \varphi}{2 \sigma_{r}^{2}}\right)
$$

Integrating this over $\mathrm{V}$ gives the probability distribution function of the phase angle, which contains the information:

$$
\begin{aligned}
P_{\varphi_{r}}\left(\varphi_{r}\right) & =\int_{0}^{\infty} P_{V_{r}, \varphi}\left(V_{r}, \varphi\right) d V=\ldots \\
\ldots & =\frac{1}{2 \pi} \exp \left(-2 S N R \sin ^{2} \varphi_{r}\right) \int_{0}^{\infty} V \times \exp \left(\frac{-\left(V-\sqrt{4 S N R} \times \cos \varphi_{r}\right)^{2}}{2} d V\right.
\end{aligned}
$$

SNR is the signal-to-noise ratio, defined as $\frac{\sqrt{E_{b}}}{N_{0}}$. When ' 1 ' $\left(\varphi=0^{\circ}\right)$ is transmitted, a 
decision error is made when the received phase angle $\varphi_{r}$ falls outside the interval of $-\frac{\pi}{2} \leq \varphi_{r} \leq \frac{\pi}{2}$, which is the decision criterion. The probability of erroneous detection then becomes:

$$
P_{M}=1-\int_{-\frac{\pi}{2}}^{\frac{\pi}{2}} P_{\varphi_{r}}\left(\varphi_{r}\right) d \varphi_{r}
$$

Note that in this final term, only the phase noise plays a role, and the amplitude noise (the $V$ terms) are cancelled out. This means that the phase detector is insensitive to noises in amplitude. As a final form, the error probability of a phase detector is given as:

$$
P_{\text {phase }}=Q\left(\sqrt{\frac{2 E_{b}}{N_{0}}}\right)=\frac{1}{2} \operatorname{erfc} \sqrt{\mathrm{SNR}}
$$

...where $Q$ is the statistical Q-function $Q(x)=\frac{1}{2 \pi} \int_{x}^{\infty} \exp -\left(\frac{u^{2}}{2}\right) d u$, and $\operatorname{erfc}(x)$ is the statistical complementary error function defined as $\operatorname{erfc}(x)=\frac{2}{\pi} \int_{0}^{\frac{\pi}{2}} \exp \left(-\frac{x^{2}}{\sin ^{2} \varphi}\right) d \varphi$. When the signal is not detected via a phase detector, but only a simple spectral evaluation was done, the amplitude noise component won't cancel out: these two orthogonal noise components will increase the unit noise energy at the detector, and therefore the probability of erroneous detection will increase as well, the following way:

$$
P_{\text {spectral }}=Q\left(\sqrt{\frac{E_{b}}{N_{0}}}\right)=\frac{1}{2} \operatorname{erfc} \sqrt{\frac{1}{2} \times \mathrm{SNR}}
$$

Figure 9 visually describes the same effect: If the phase angle of the signal is analysed instead of the magnitude, detection reliability can be increased.

\section{Using many trials and phase coherency}

The method described above works for single trials, but in many cases particularly with human EEG or long distance radio communication, the signal-to-noise ratio is just too low to produce a meaningful result. Provided that the system under test does not 
change fundamentally, the remedy for this problem is to collect data from many trials, and analyse the mean values across trials. In communication engineering, the equivalent method is to use more than one channel to receive the same signal at the same time, by using a diversity receiver, or to use repetition coding [Proakis, 2000]. If the system under test does not change fundamentally during the frequency tagging experiment, it is reasonable to assume that the phase shift $d \varphi$ and the gain of the system $g_{n}$ are constant. If this is the case, it is possible to discard the phase information along with its noise component from the data set, and analyse the confidence interval of the collected phase angles rather than the mean value of the phase angles themselves. The analysis of the confidence interval of the phase angles is the phase coherency analysis, which was introduced by Norcia and Tyler in 1984, and they derived it from first principles [Norcia and Tyler, 1984] [Tyler and Kaitz, 1977]. Having established above that an experiment with many collected trials can be considered to be a multi-channel system with the same signal transmitted in each trial irrespective whether the trials are spatially or temporally distributed, we can modify the probability equations to incorporate many channels or trials. In the previous section we have shown the probability of detection errors of phase-modulated signals as a function of signal-to-noise ratio:

$$
P_{\text {phase }}=Q\left(\sqrt{\frac{2 E_{b}}{N_{0}}}\right)
$$

Following the proof for the quadratic equation of the decision threshold in [Proakis and Salehi, 2008], the SNR is expressed as:

$$
\mathrm{SNR}=\frac{E_{b}}{N_{0}} \sum_{n=1}^{L}\left|g_{n}\right|^{2}
$$

...where the experiment was conducted across $L$ number of trials, and the gain of the system for each channel $g_{n}$ is known. In practice $g_{n}$ can vary somewhat during an experiment: some EEG electrodes can change impedance throughout the experiment, 
or a radio signal can fade due to a change of atmospheric conditions, for example. In EEG frequency tagging applications, these impedances are kept at a relatively constant level throughout the recording, and are given in studies

[Norcia and Tyler, 1984, Cottereau et al., 2014, Jamison et al., 2015], or set to a recommended level by the EEG set's manufacturer during the application of the electrode cap. Therefore, we can assume that from trial-to-trial, the channel gain $g_{n}$ is not going to be significantly different.

Then, the SNR becomes:

$$
\mathrm{SNR}=L \times \frac{E_{b}}{N_{0}}|g|^{2}
$$

The gain of the system is going to purely depend on the system under test. Therefore, the $P$ value of the phase distribution tested against an other phase distribution built up from Gaussian noise is going to be:

$$
P_{\varphi}=Q(\sqrt{2 \times L \times \mathrm{SNR}})=\frac{1}{2} \operatorname{erfc} \sqrt{2 \times L \times \mathrm{SNR}}
$$

Using a multi-channel system therefore boosts the signal to noise ratio, and the more trials used in an experiment, it is possible to obtain ever more significant results. Inter-trial coherency is a related measure, which does not reveal the phase angle $\varphi$ itself. Since we only transmit a single bit of information for each trial, the exact quantity of $\varphi$ is not required. If we can reliably establish that the temporal modulation frequency of interest $\hat{V}_{f}$ 's inter trial coherency is significantly higher than the the inter-trial coherency calculated from a Gaussian noise distribution created from the same number of samples, we have detected the signal. Across $L$ number of trials the coherency calculated as:

$$
\operatorname{coh}(f)_{L}=\left|\left\langle\frac{\hat{V}(f)}{|\hat{V}(f)|}\right\rangle_{L}\right|
$$

In practice, to convert between the time and frequency domains, the Fourier transform is used. For a chosen frequency, the Fourier-coefficients are averaged across $L$ good 
trials as complex numbers, to produce the vector means. Then, the magnitudes of each Fourier-coefficient at the frequency $f$ are averaged across $L$ trials to produce the scalar means. When the corresponding Fourier-coefficient's vector means are divided by the scalar means, the $\operatorname{coh}(f)_{L}$, the inter-trial coherency is calculated across $L$ trials. This operation produces a scalar between 0 and 1, and this process can be repeated for a a selected temporal frequency as it was done in Norcia and Tyler's study [Norcia and Tyler, 1984], or to all the Fourier-coefficients in the entire spectrum. An alternative method to calculate coherency is to look at the distribution of the phase angles of a single coefficient:

$$
\operatorname{coh}(f)_{L}=\frac{2 \pi-C I_{\varphi_{[L]}}}{2 \pi}
$$

...where $C I_{\varphi_{[L]}}$ is the confidence interval of the phase angle distribution across the trials, as shown in figure 3 .

The calculated coherency value then tells that proportionally how much of the corresponding Fourier-coefficient's phase angle was the same from trial to trial.

Therefore, theoretically, white noise has zero coherency, and a sinewave that is exactly in the same phase in every trial, will have a coherency of 1

[Norcia and Tyler, 1984, Tyler and Kaitz, 1977].

The probability distribution of phase angles $P_{\varphi}$ tells us about what is the probability of a randomly selected data point in the distribution to be outside the decision threshold. In the binary signal representation introduced here, the decision threshold was $-\frac{\pi}{2} \leq \varphi_{r} \leq \frac{\pi}{2}$ radians. In coherency, since magnitude of the phase angle $\varphi$ is not used, it is possible to say that the coherency detects the presence of the coherent signal at the receiver. While the P-value of the phase distribution is clearly related to the coherency distribution, the theory suggests that it is impossible to have 'unary' signals:

$$
\lim _{M \rightarrow 1} P_{P S K}=\frac{1}{2} \operatorname{erfc}\left(\sqrt{\frac{2 \times L \times S N R}{\log _{2} M}}\right)=0
$$


However, despite the 'approximate' nature of this function [Proakis and Salehi, 2008], diverging away from the binary system $(M=2)$ towards 'unary' $(M=1)$ shows that the P-values decrease, suggesting that the transmission quality will increase. Therefore, by using inter-trial coherency detecting the presence of a carrier that is always in the same phase in all the trials, we have a much better chance of detecting a weak signal, such as the carriers hidden in frequency-tagged EEG.

From the previous section, we have seen that the erroneous detection probability of spectral evaluation as a function of signal-to-noise-ratio and the number of trials used is:

$$
P_{\text {spectral }}=\frac{1}{2} \operatorname{erfc} \sqrt{\frac{1}{2} \times \mathrm{SNR} \times \sum_{n=1}^{L}\left|g_{n}\right|^{2}}
$$

Phase analysis on stimuli with annotated phase information, yield lower probability under the same conditions:

$$
P_{\text {phase }}=\frac{1}{2} \operatorname{erfc} \sqrt{\mathrm{SNR} \times \sum_{n=1}^{L}\left|g_{n}\right|^{2}}
$$

If it is possible to keep the phase angle constant over many trials, coherency gives the lowest error probability. However, the advantage can only be estimated:

$$
P_{\text {coherency }} \approx \frac{1}{2} \operatorname{erfc} \sqrt{2 \times \mathrm{SNR} \times \sum_{n=1}^{L}\left|g_{n}\right|^{2}}
$$

...where $\operatorname{erfc}$ is the complementary error function, SNR is the signal to noise ratio, $g_{n}$ is the gain of the system and $L$ is the number of trials. Conversely, for each modulation method, the minimum number of trials required to achieve a $P=0.05$ can be calculated as:

$$
L_{\text {spectral }}=\frac{\left.\left[\operatorname{erfc}^{-1}(2 \times 0.05)\right]^{2}\right]}{\mathrm{SNR}}
$$

$$
L_{\text {phase }}=\frac{\left.\frac{1}{2} \times\left[e r f c^{-1}(2 \times 0.05)\right]^{2}\right]}{\text { SNR }}
$$




$$
L_{\text {coherency }} \approx \frac{\left.\frac{1}{4} \times\left[e r f c^{-1}(2 \times 0.05)\right]^{2}\right]}{\operatorname{SNR}}
$$

\section{Figures}

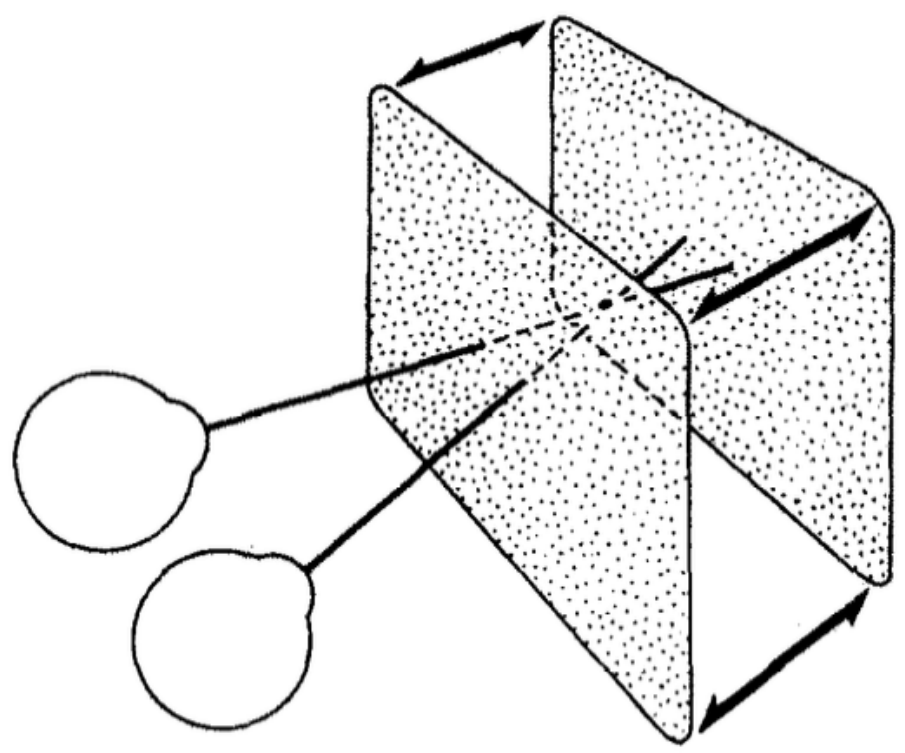

Figure 1: The stimulus shown in the example: the dots defined a plane, which then jumped in front of and behind the screen, at a set temporal frequency [Norcia and Tyler, 1984]. This elicited a signal in our participants, which we could detect in their EEG recordings. 

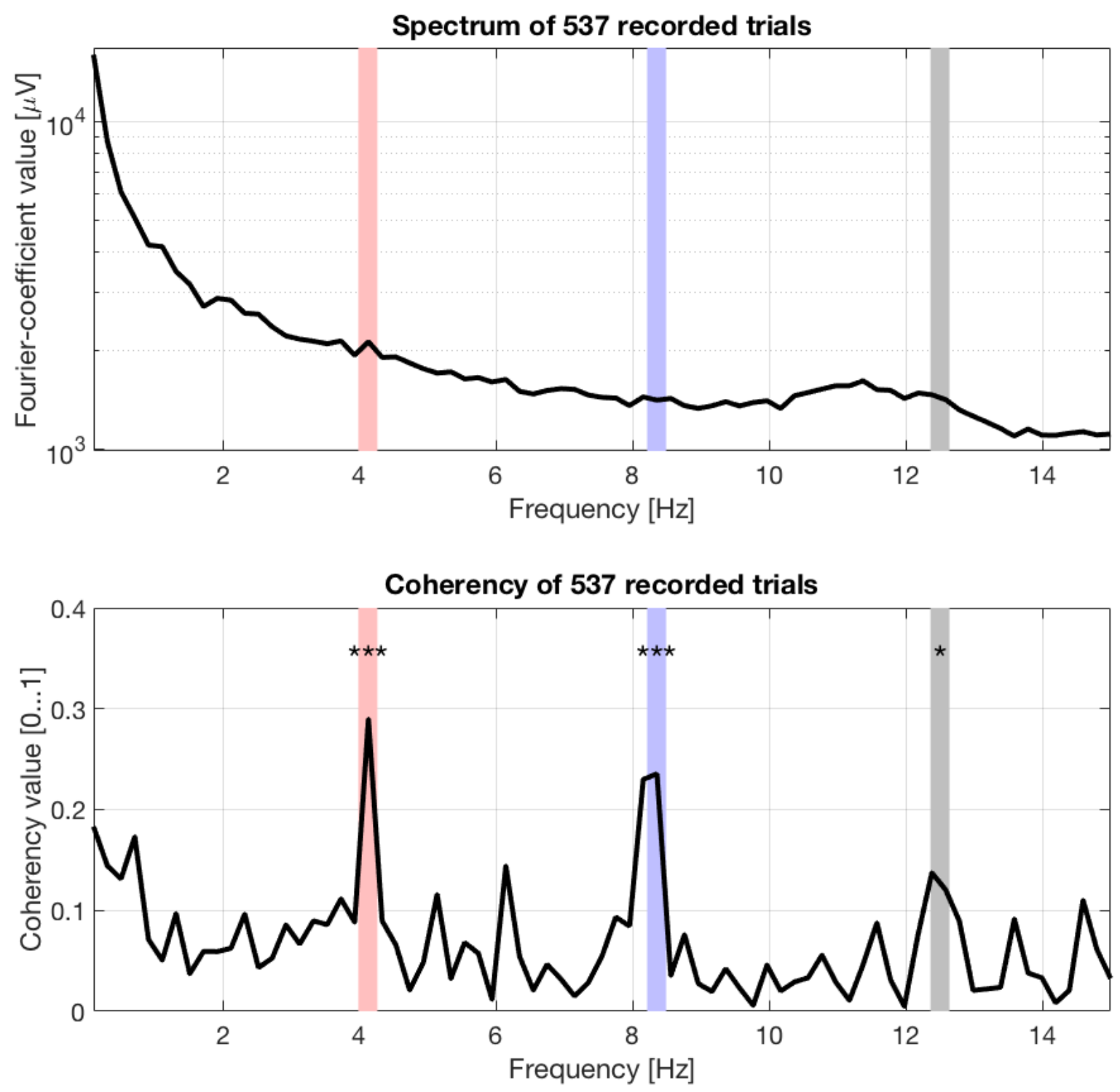

Figure 2: The analysis was performed on the same data in two different ways: the top plot shows the result of averaging in the frequency domain. When we plot the inter-trial coherency of the same data, the results show significantly increased coherency at the second $(4.2 \mathrm{~Hz}$. red area), fourth $(8.4 \mathrm{~Hz}$, blue area) and sixth $(12.6 \mathrm{~Hz}$, grey area) harmonics of the signal. 

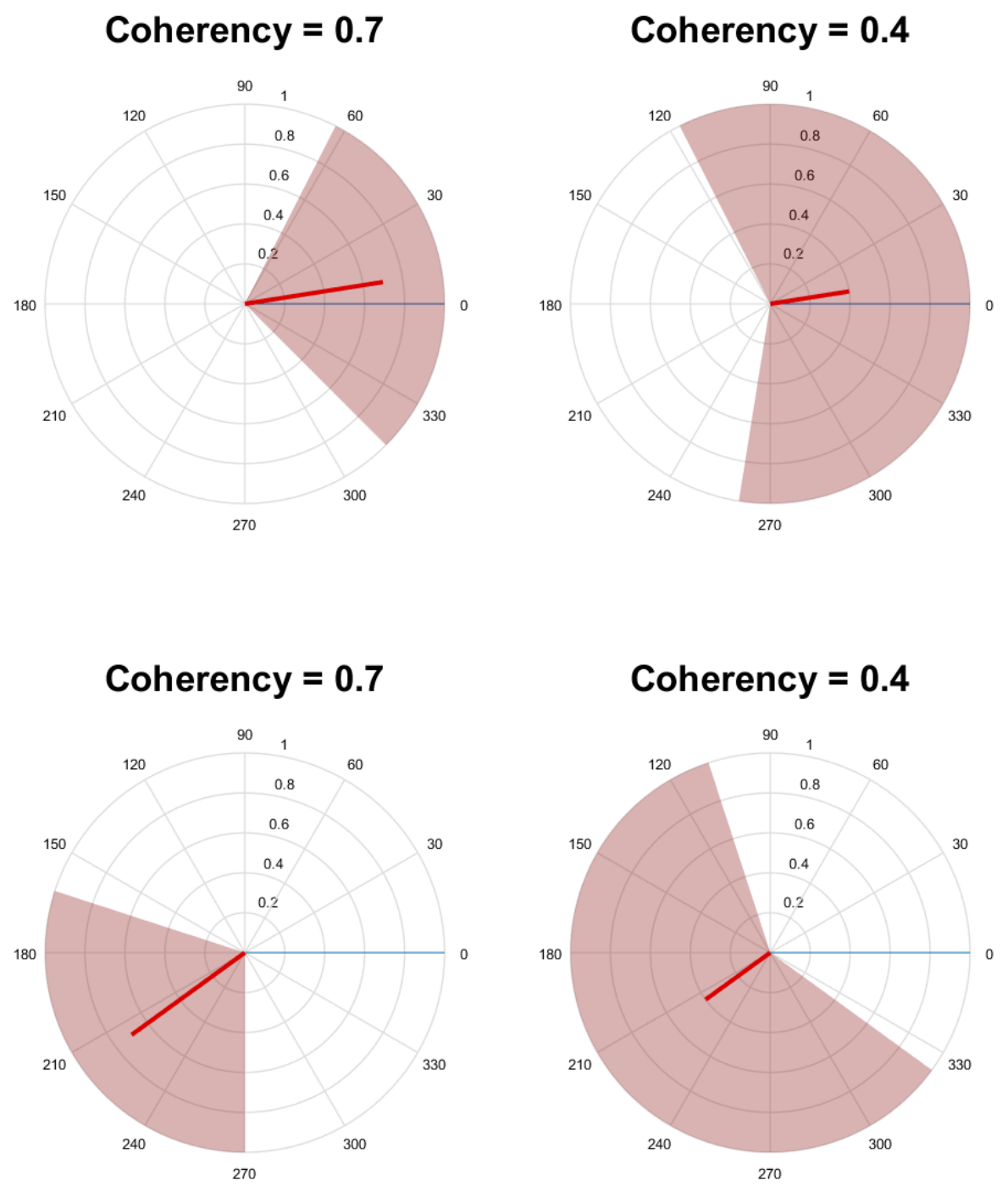

Figure 3: When looking at coherency, we are looking at the distribution of vectors across the $L$ trials. The coherency is inversely proportional to the $95 \%$ confidence interval of this vector distribution (shaded area), and does not include information about the phase angle indicated with the vector. 

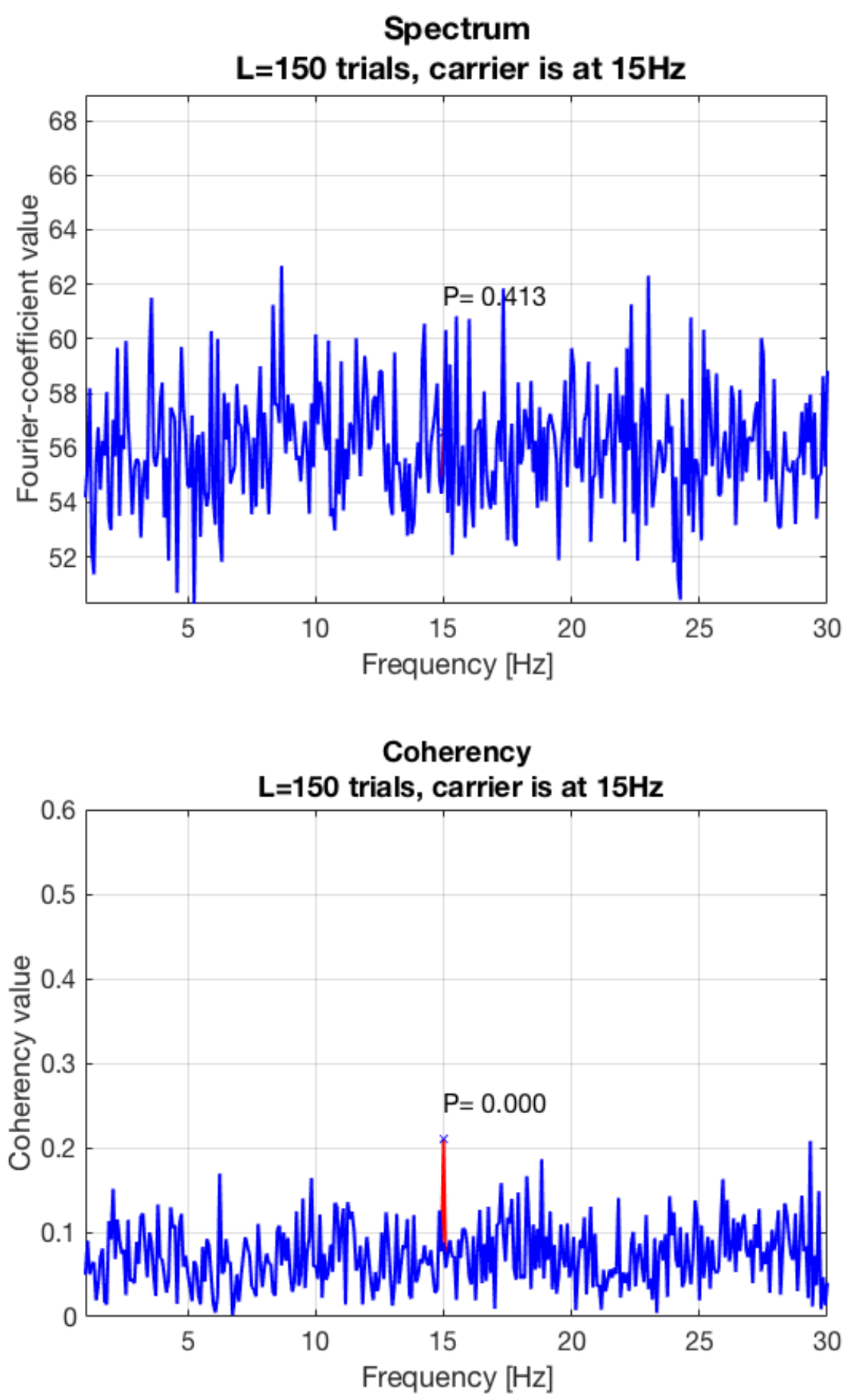

Figure 4: $A$ weak signal $\left(S N R=\frac{1}{300}\right)$ at $f=15 \mathrm{~Hz}$ can not be detected using the spectral analysis method in the top plot. However, provided that the phase of the signal was the same from trial to trial, the coherency analysis method confidently detects the signal at the same SNR in the bottom plot. 

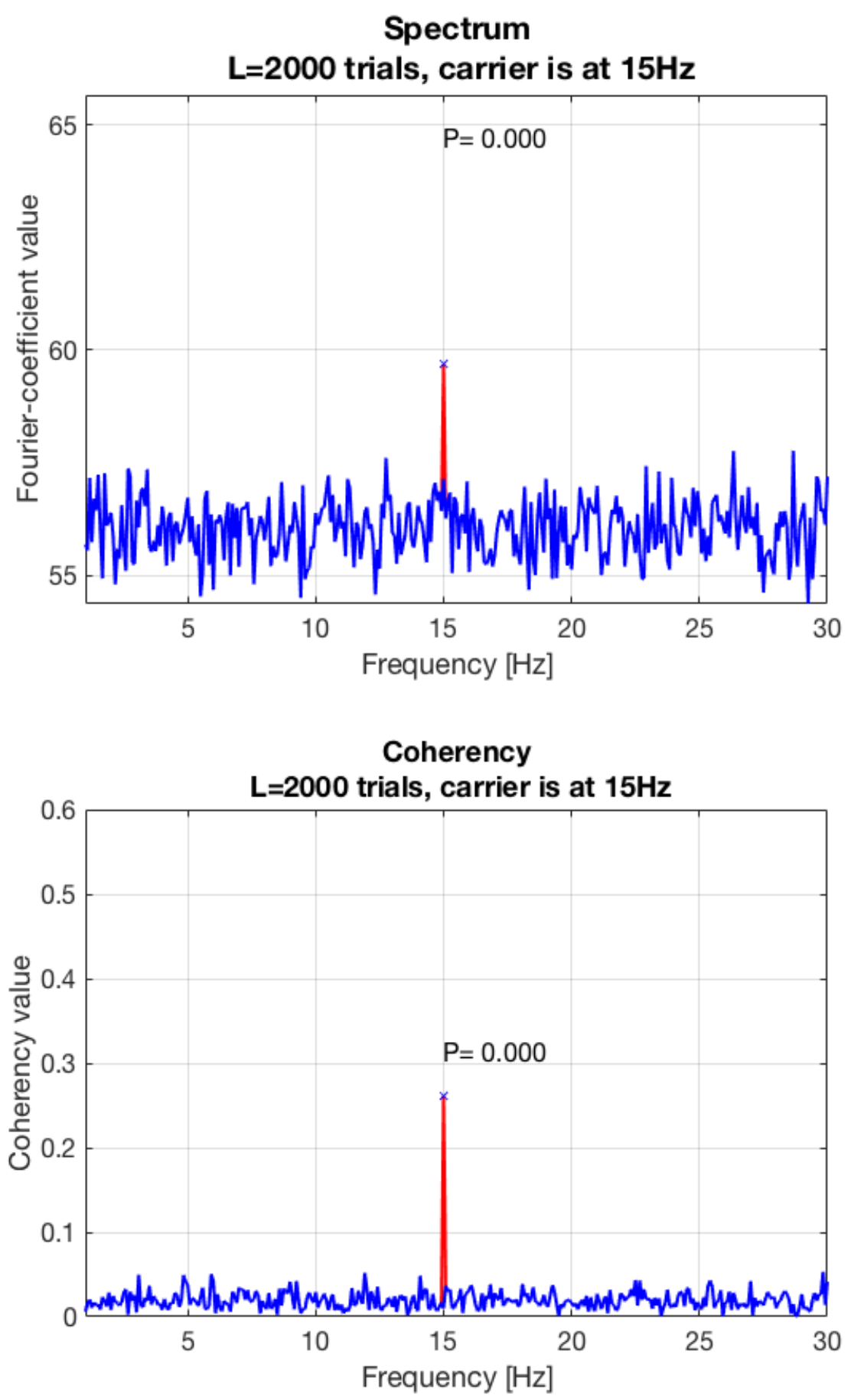

Figure 5: At 2000 trials, a weak signal at $f=15 \mathrm{~Hz}$ with the SNR of $\frac{1}{300}$ can be detected with both the spectral analysis in the top plot, and the coherency analysis at the bottom plot. 


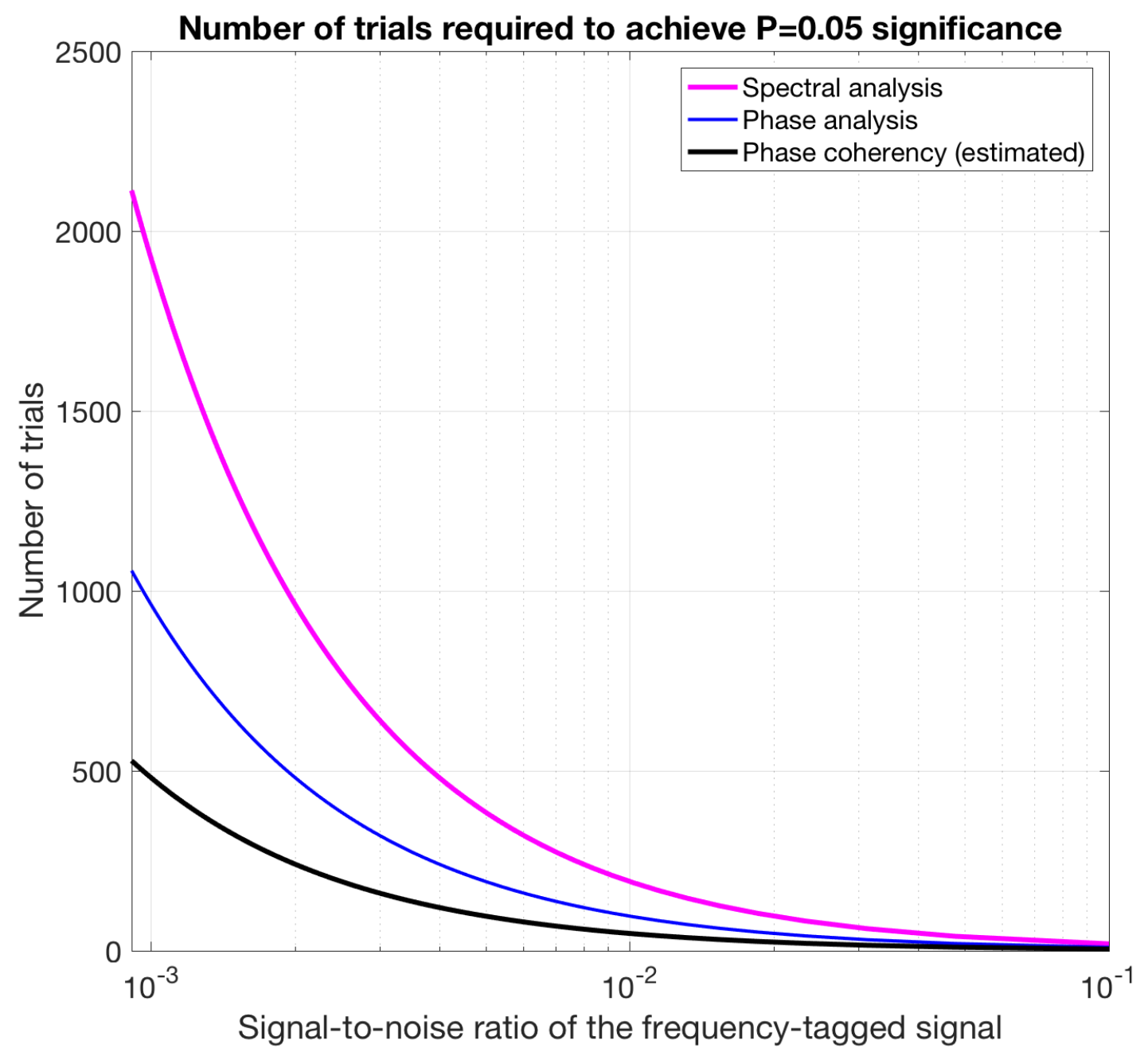

Figure 6: Inter-trial (phase) coherency requires the least number of trials for a given signal-to-noise ratio. 
B
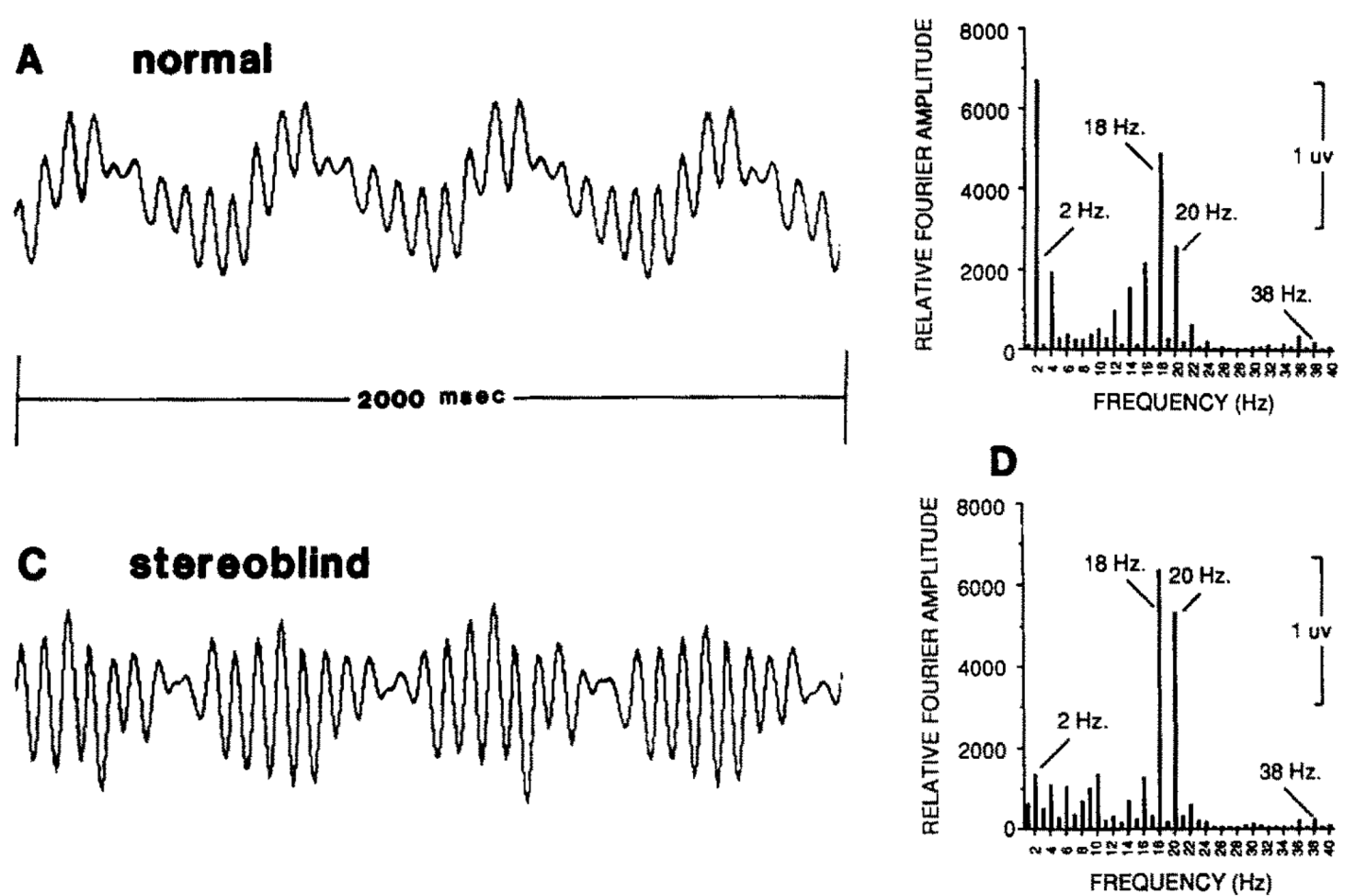

Figure 7: An example of two-carrier frequency tagging with human EEG: When flashes with different temporal frequencies are presented at each eye, stereoblind people do not produce the intermodulational products of the two frequencies [Baitch and Levi, 1988]. 


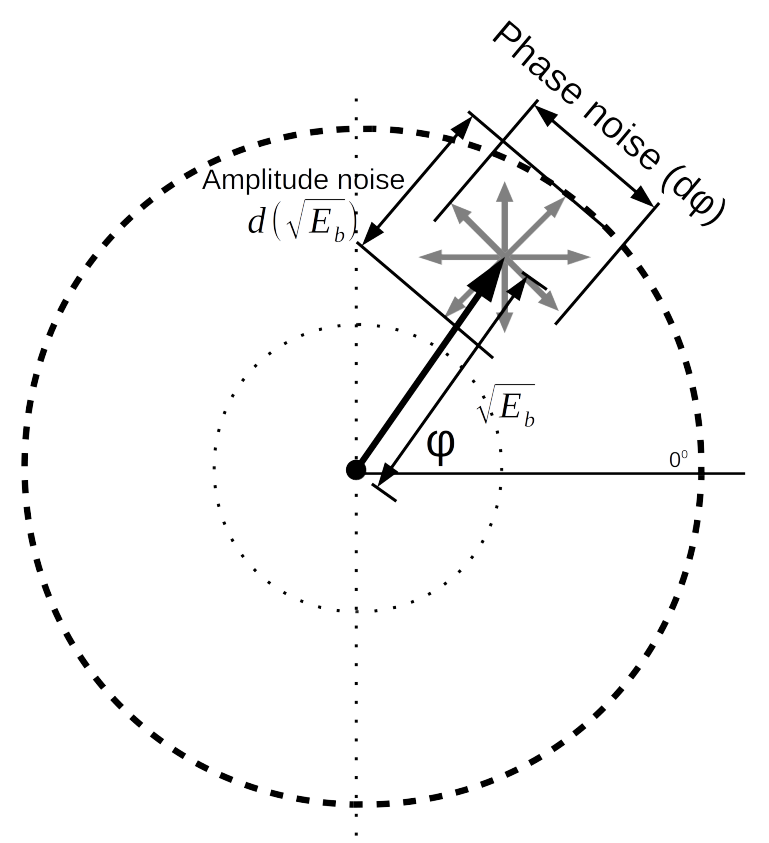

Figure 8: The received signal at the tagged frequency expressed with a vector. The role of the noise components $d \sqrt{E_{b}}$ and $d \phi$ are indicated with the arrows at the end of the vector. 

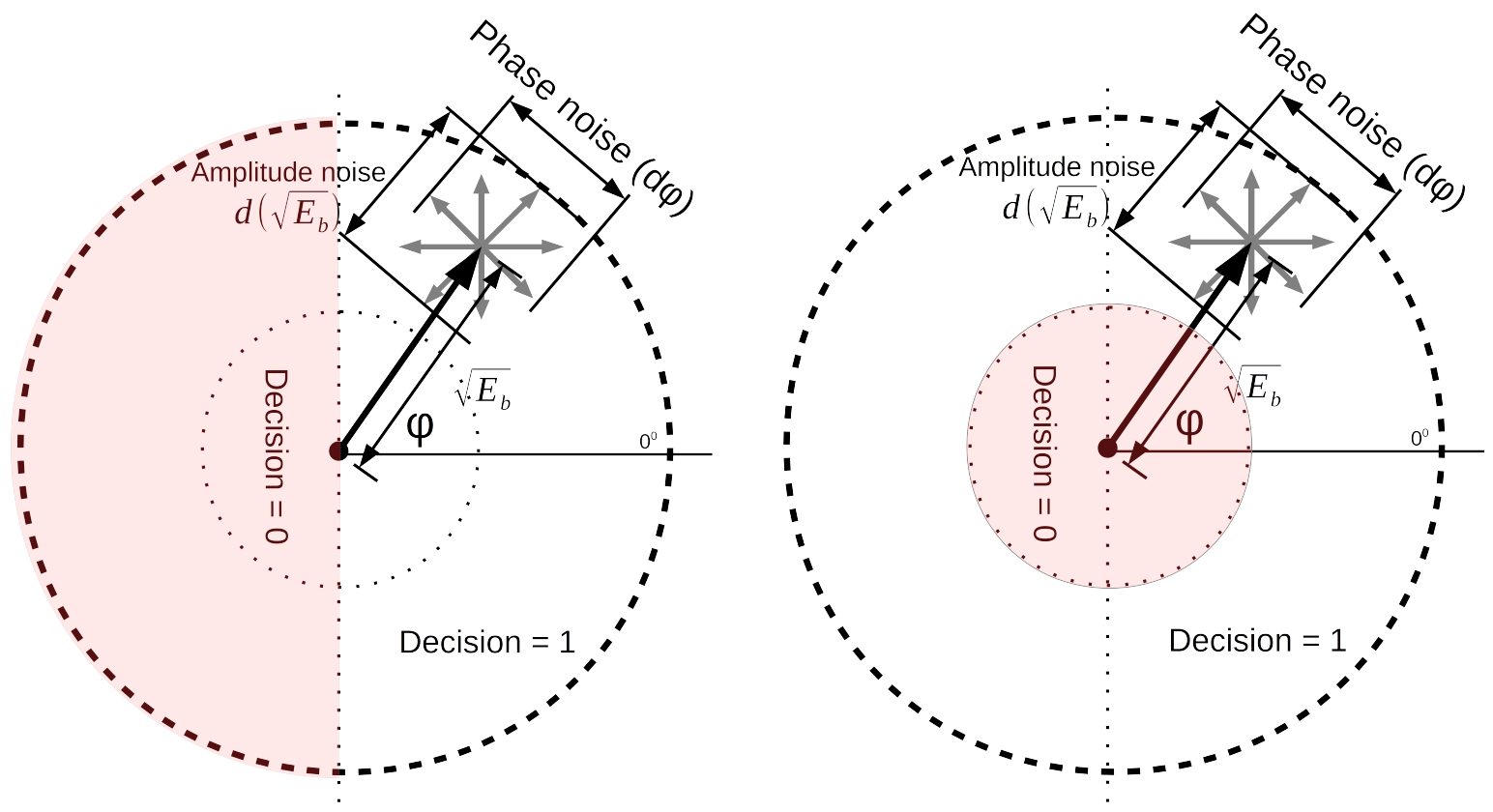

Figure 9: Left plot: Provided that the phase of the frequency-tagged signal is known, it is possible to reject the amplitude noise component $d \sqrt{E_{b}}$, and detect the signal using only its phase angle: if the signal's phase angle is outside the shaded area, the signal is detected. Right plot: When spectral analysis is used, both noise components play a role. If the vector's end is outside the central shaded area, the signal is detected.

Note that as the signal-to-noise ratio decreases, the central shaded area generated by the noise in the right plot increases, making detection of the signal increasingly more difficult. However, the shaded area of phase angles in the right plot stays constant, as it is independent of the amplitude noise component, clearly showing the advantage of the phase analysis method. 


\section{${ }^{{ }_{30}}$ References}

[Baitch and Levi, 1988] Baitch, L. W. and Levi, D. M. (1988). Evidence for nonlinear binocular interactions in human visual cortex. Vision research, 28(10):1139-1143.

[Boremanse et al., 2013] Boremanse, A., Norcia, A. M., and Rossion, B. (2013). An objective signature for visual binding of face parts in the human brain. Journal of Vision, 13(11):6-6.

[Ciganek, 1961] Ciganek, L. (1961). The eeg response (evoked potential) to light stimulus in man. Electroencephalography and clinical Neurophysiology, 13(2):165-172.

[Cottereau et al., 2014] Cottereau, B. R., McKee, S. P., and Norcia, A. M. (2014). Dynamics and cortical distribution of neural responses to $2 \mathrm{~d}$ and $3 \mathrm{~d}$ motion in human. Journal of neurophysiology, 111(3):533-543.

[Daubechies, 1990] Daubechies, I. (1990). The wavelet transform, time-frequency localization and signal analysis. Information Theory, IEEE Transactions on, 36(5):961-1005.

[Delorme and Makeig, 2004] Delorme, A. and Makeig, S. (2004). Eeglab: an open source toolbox for analysis of single-trial eeg dynamics including independent component analysis. Journal of neuroscience methods, 134(1):9-21. 
[Jamison et al., 2015] Jamison, K. W., Roy, A. V., He, S., Engel, S. A., and He, B. (2015). Ssvep signatures of binocular rivalry during simultaneous eeg and fmri. Journal of neuroscience methods, 243:53-62.

[Julesz, 1971] Julesz, B. (1971). Foundations of cyclopean perception.

[Moungou et al., 2016] Moungou, A., Thonnard, J.-L., and Mouraux, A. (2016). Eeg frequency tagging to explore the cortical activity related to the tactile exploration of natural textures. Scientific reports, 6.

[Norcia et al., 2015] Norcia, A. M., Appelbaum, L. G., Ales, J. M., Cottereau, B. R., and Rossion, B. (2015). The steady-state visual evoked potential in vision research: a review. Journal of vision, 15(6):4-4.

[Norcia and Tyler, 1984] Norcia, A. M. and Tyler, C. W. (1984). Temporal frequency limits for stereoscopic apparent motion processes. Vision research, 24(5):395-401.

[Proakis, 2000] Proakis, J. G. (2000). Digital communications, fourth edition.

[Proakis and Salehi, 2008] Proakis, J. G. and Salehi, M. (2008). Digital communications, fifth edition.

[Tyler and Kaitz, 1977] Tyler, C. and Kaitz, M. (1977). Movement adaptation in the visual evoked response. Experimental Brain Research, 27(2):203-209. 